more active ingredients in the powder without significantly affecting the internal structure.

The rhombohedral modification completely transforms to common hexagonal after it has been reacted with bromine or potassium and produces lamellar compounds. The transformation involves periodic slip of hexagonal layers by $a / 3$. Accompanied by the rearrangement, it is likely that $a$ slight release of the rotational misorientation of layers may occur and result in the narrowing of (1122) line. Boehm and Hofmann observed that on treatment with bromine partial recovery of the distortion, that is, the extent in the sample obtained by $72 \mathrm{~h}$ grinding, decreased to that of $20 \mathrm{~h}$ of grinding.

Using the oxidized graphite, I have carried out a series of experiments with this objective: but the results have not been conclusive. Sometimes definite narrowing was observed in the case of ground powders, but this is considered to be due to the removal of the less-stable components in the powder by some causes, which has been suggested by a rapid decrease in the breadth of the (11 $\overline{2} 2)$ line at the beginning of the boiling with the reagent attaining an ultimate breadth of about 90 per cent of the starting powder. The treatments with bromine and potassium were performed as follows: (1) the powder was exposed to bromine vapour for 2 weeks at room temperature and then evaporated at $150^{\circ} \mathrm{C} ;(2)$ the powder was immersed in a mixture of 1 part of bromine and 3 parts of carbon tetrachloride for 2 weeks at room temperature and then evaporated at $150^{\circ} \mathrm{C}$; (3) amalgamated with potassium metal at $150^{\circ} \mathrm{C}$ and then evaporated at $500^{\circ} \mathrm{C}$ for 3 days; (4) amalgamated with potassium metal at $150^{\circ} \mathrm{C}$ and then partially evaporated at $450^{\circ} \mathrm{C}$ for $10 \mathrm{~h}$. The procedure was repeated ten times and finally evaporated at $500^{\circ} \mathrm{C}$ for 3 days.

Faculty of Engineering,

Yamaguchi University, Ube, Japan.

I Bacon, G. E., Acta Cryst., 3, 320 (1950).

2 Bacon, G. E., Proc. Third Carbon Conf., 475 (Pergamon Press, 1959).

' Boehm, H. F., and Hofmann, U., Z. anorg. allgem. Chem., 278, 58 (1955).

\section{Crystal Structure of Imidazol}

$W_{\mathrm{E}}$ have undertaken an analysis of the crystal structure of imidazol $\left(\mathrm{C}_{3} \mathrm{~N}_{2} \mathrm{H}_{4}\right)$. The space group is $P 2_{1} / c$ instead of $P 2 / m$ as reported by Greenwood ${ }^{1}$, with the $c$-axis doubled. The cell dimensions are $a=7 \cdot 70 \AA, b=5.43 \AA, c=$ $9 \cdot 73 \AA, \beta=117^{\circ} 20^{\prime}$, with four molecules per unit cell.

The structure was solved in three projections, deconvoluting the sharpened Patterson projections by convolu-

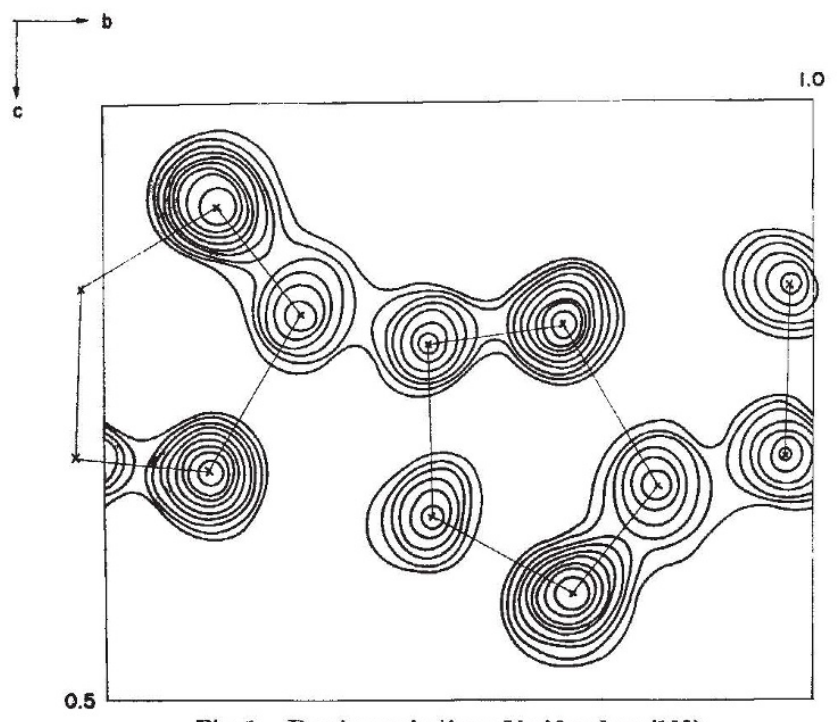

Fig. 1. Fourier projection of imidazol on (100) tion molecule methods ${ }^{2}$. This approach lod to an initial parameter set, which could be refined with least squares calculations in the combined projections together with Fourier projections. The electron density of the structure in projection on (100) is shown in Fig. 1. The $R$-factor at present is $19 \cdot 3$ per cent in the combined projections with co-ordinates, as listed in Table 1. Accuracy is limited by the crystals, which could only be grown as fibrous needles of poor quality. Further, due to the hygroscopicity of imidazol it was necessary that the crystals for diffraction investigations be sealed in glass capillaries.

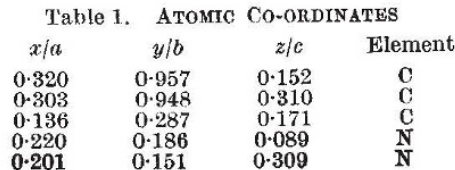

The molecules are found with the molecular plane nearly perpendicular to $(001)$, forming an angle $\pm 32^{\circ}$ with $[010]$. The Fourier maps indicate hydrogen bridges along the $c$-axis, connecting two molecules over the imino hydrogen, with an $\mathrm{N}-\mathrm{H}$. . . N distance between two molecules of $2.7 \AA$. All the other intermolecular distanees are greater than $3 \cdot 1 \AA$.

The molecules connected by hydrogen bridges are twisted against each other by $64^{\circ}$, forming chains along the $c$-axis. The fibrous appearance of the erystals, with the fibre axis parallel to the $c$-axis, can be explained by this finding.

GEORG WiLI

U.S. Army Electronics Research and Development

Laboratory, Fort Monmouth, New Jersey.

1 Greenwood, G., Min. Mag.,21, 1 (1926).

${ }^{2}$ Will, G., Z. Krist. (in the press).

\section{METALLURGY}

\section{Incidence of Grain Boundary Cracking in Creep Fracture}

Is a recent investigation of void nucleation ${ }^{1}$ the incidence of cavitation was determined metallographically by an examination of longitudinal sections of fractured specimens. Lines were drawn parallel and at right angles to the direction of the applied stress, and all grain boundaries intersected by these lines were separated into three groups depending on the angle made on the polished section of the specimen by the grain boundary with the tensile axis. For each anglo interval $\left(0^{\circ}-30^{\circ}, 30^{\circ}-60^{\circ}\right.$, $60^{\circ}-90^{\circ}$ ), a count was made of the number of boundaries with and without cavities located on them. The work was carried out on a dilute nickel alloy (containing $0 \cdot 1$ atomic per cent gold) and the incidence of cavitation measured over a limited range of tensile creep stresses. Over this narrow range, the crack incidence appeared to be independent of the applied tensile stress. It was therefore decided to apply the same method of assessing crack incidence over a wider stress range.

The present work was carried out at $500^{\circ} \mathrm{C}$ using pure nickel (99.97 per cent). Details of the material, specimen preparation and creep testing procedure have been given elsewhere ${ }^{2}$. The tensile creep stresses used were 8,6 and 3.5 tons/in. ${ }^{2}$ which gave rupture lives of 109,320 and $16,500 \mathrm{~min}$ respectively. Longitudinal sections of the fractured test-pieces were lightly etched and the incidence of cavitation determined. More than 500 grain boundaries were examined in each specimen. The results are given in Table 1. It would appear that in each angle interval the

Table 1. No. OF CRACKeD BoUndaries IN ANY ANGLE INTERVAI RXPRESSED as a Pfircentage of the Total No. OF Boundarizs in that INTervat $\begin{array}{cccc}\text { Stress (tons/in. }{ }^{2} \text { ) } & 90^{\circ}-60^{\circ} & 60^{\circ}-30^{\circ} & 30^{\circ}-0^{\circ} \\ 8 & 45 & 29 & 7 \\ 6 & 43 & 31 & 7 \\ 3.5 & 47 & 30 & 5\end{array}$ 